\title{
ASYMMETRIES AROUND LUMINOUS RED VARIABLES
}

\author{
ANTONIO MÁRIO MAGALHÃES \\ Instituto Astronômico e Geofísico, Universidade de São Paulo \\ São Paulo, Brazil
}

AND

KENNETH H. NORDSIECK

Space Astronomy Laboratory, University of Wisconsin Madison, WI 53706, U.S.A.

\begin{abstract}
Departures from spherical symmetry are known to exist around Luminous Red Variables (LRVs). One of the earliest pieces of evidence came from the discovery that light from these objects is polarized. Additional evidence comes from other techniques and observations of related objects. This talk briefly reviews these data and focuses on the mechanisms that produce polarization in LRVs. Emphasis is given on recent spectropolarimetric monitoring data of selected key objects and what we have learned about the LRV environment from such data.
\end{abstract}

\section{Introduction}

Mass loss plays a central role in the late stages of stellar evolution. In addition, mass loss from Asymptotic Giant Branch (AGB) stars, such as Mira variables, and from red supergiants may be responsible for up to $60 \%$, relative to all stars, of the interstellar dust input into the interstellar medium (Gehrz 1989). Mass loss for low and intermediate mass stars occurs at rates of up to $10^{-4}$ solar masses/yr in the AGB stage (see, e.g., Chapman 1994 for a review). It has been proposed (e.g. Bedijn 1988; Bowen \& Willson 1991) that the mass-loss rate actually increases with time so that a star loses a good share of its mass near the end of the AGB evolution, its envelope being finally stripped away.

Despite the success of this general picture, details of the physical processes occurring during mass loss are still not well understood. Models for 
pulsating red giant stars have been able to incorporate a great deal of physics in describing the atmospheric structure and stellar winds from Luminous Red Variables, or LRVs (Bowen \& Willson 1991). However, these impressive models assume spherical symmetry, despite the fact that departures from spherical symmetry are known to exist around LRVs. One piece of evidence for this is that light from these objects typically shows some degree of linear polarization.

Intrinsic optical polarization in red long-period variables was discovered in the early 1960s (see Magalhães 1988 for a review) and indicated that nonspherically-symmetric structures do exist in the extended atmospheres of LRVs (see §2). Further evidence of asphericity in LRV envelopes comes from details of OH maser emission profiles (Collison \& Fix 1992), K I resonant scattering (Plez \& Lambert 1994), rings of $\mathrm{SiO}$ maser emission (Diamond et al. 1994; Greenhill et al. 1995) and OH radio images (Chapman et al. 1994). Aspherical symmetries such as found by Trammell, Dinerstein \& Goodrich (1994) for post-AGB stars from spectropolarimetry may then be naturally understood, as they are already present in earlier stages. The observed, more obvious non-spherical symmetries in Protoplanetary and Planetary Nebulae (PPN) studied in the optical and IR (Kwok 1993) and also seen in optical polarimetry of evolved objects (Johnson \& Jones 1991; Trammell et al. 1994) are also consistent with the origin of the asymmetries being early in the AGB phase.

\section{Mechanisms that Produce Polarization in LRVs}

Polarization observations yield otherwise unobtainable diagnostics related to the geometrical structure of unresolvable objects as well as the nature of the scattering physics. The degree of polarization depends on the nature and optical depth of the scatterers and on their geometrical distribution. The polarimetric wavelength dependence arises from the wavelength dependence of single-scattering polarization, of the scattering cross-section, of any competing opacity, and of any unpolarized, diluting stellar light. Time dependence arises when the asymmetry or the optical depth of the scatterers varies with time, as in a pulsating star or variable stellar wind.

The degree of linear polarization $P$, in percentage, and the position angle $\theta$ are usually transformed for analysis into two of the Stokes parameters, $Q=P I \cos (2 \theta)$ and $U=P I \sin (2 \theta)$, where $I$, the first Stokes parameter, is the beam intensity (the fourth Stokes parameter is $V$, which describes circular polarization). In the $Q-U$ plane, the polarization is represented by polar coordinates $\left\{\left[Q^{2}+U^{2}\right]^{1 / 2}, 2 \theta\right\}$. If the observed polarization results from multiple components (e.g. intrinsic plus interstellar), the $Q, U$ components of each polarization add like a vector at each wavelength. Since the 
polarization is usually small, this will also be true for the more often used normalized Stokes parameters, $q=Q / I$ and $u=U / I$.

In general, the optical linear polarization of LRVs is moderately large (a few to several percent); the polarization position angle and wavelength dependence typically vary with time, and the polarization typically increases with decreasing wavelength, which is taken as indicative of Rayleigh or Mie scattering. Two basic models have been invoked to explain the observations. One of them involves scattering in a non-spherically-symmetric dust cloud (Kruszewski, Gehrels \& Serkowski 1968; Shawl 1975). Shawl (1975) has considered optically thin models whereas the Monte Carlo method can be used for arbitrary optical depths (Lefêvre \& Daniel 1988).

In contrast, photospheric scattering, coupled to some asymmetry over the stellar disk, may cause the observed polarization (Harrington 1969). The strong gradient of the photospheric source function with optical depth at the shorter wavelengths will cause radiation at these wavelengths to come predominantly from deeper layers. Due to molecular Rayleigh scattering, the phase function of which has maximum polarization at a $90^{\circ}$ degree scattering angle, this may cause strong limb polarization, with position angle usually tangent to the limb.

The needed asymmetries over the stellar disk may arise from nonradial pulsations, stellar spots, or other, more systematic variations in the temperature across the stellar surface. Temperature differences could indeed result from the formation of giant convective cells (Schwarzschild 1975). Origins for asymmetries in models for the atmospheric structure of LRVs are further discussed by Willson \& Bowen (1988). The wavelength dependence of the polarization produced by an asymmetric photosphere will depend on how the ratio of absorption to scattering varies with optical depth and wavelength and the temperature gradient (Magalhães, Coyne \& Benedetti 1986). A combination of stellar surface asymmetries with scattering by a dust shell could also give rise to a net polarization. Changes in the polarization across molecular bands may also result from dust scattering alone, as the size of the source as seen by the dust shell will vary with wavelength across the feature. A large stellar spot, with a different overall temperature than the remainder of the stellar disk, may provide the asymmetry and cause changes in the polarization across spectral features.

Finally, circular polarization may also appear in LRVs from multiple Mie scattering in an asymmetric dust envelope or magneto-emission from stellar spots (Holenstein 1993). Multiple scattering by molecules per se will cause no circular polarization. Light leaving the photosphere linearly polarized may be circularly polarized when scattered off a dust envelope. 


\section{Spectropolarimetric Monitoring of LRVs}

The accurate detection of changes in the polarization across photospheric features and with time should thus provide a wealth of information concerning the processes occurring in the LRV environment and help distinguish among different models (Magalhães 1988). Since 1989, a dedicated spectropolarimeter, HPOL, has been operating on every clear night at the University of Wisconsin's 90-cm telescope at Pine Bluff Observatory (PBO). Its main features are high polarimetric stability $(0.002 \%$ in broad band work), high precision due to the large dynamic range of the CCD, and wide wavelength coverage (presently $3200-10500 \AA$, with $8 \AA$ resolution). We have been monitoring a few specific red variables which earlier data have shown to be of particular interest. A survey of several red variables is also being conducted. Below we discuss some of the data for three red variables, namely $\alpha$ Ori, V CVn and $o$ Cet (Mira), which are among the ones with better phase coverage. These data are currently being prepared for publication and give further clues as to the processes involved in causing polarization and the diverse phenomena that may take place in the complex circumstellar environments of LRVs.

\section{1. $\alpha$ Ori}

Alpha Ori is a red supergiant of type M2 Iab, with probably around 10 solar masses, 1350 solar radii and $3500 \mathrm{~K}$ effective temperature. It is a semiregular variable of type SRc.

Alpha Ori has been monitored spectropolarimetrically at PBO since 1990. In December 1990 and February 1995 it was also observed from $1500 \AA$ to $3000 \AA$ by the Wisconsin Ultraviolet Photo Polarimeter Experiment, WUPPE, on board the Space Shuttle Astro-1 and Astro-2 observatories. In the PBO data, the different TiO band systems $\left(\gamma, \gamma^{\prime}\right.$ and $\left.\alpha\right)$ show distinct behavior in percent polarization and position angle. Further, "B filter" polarization measurements (obtained from the spectral data) and the TiO $\gamma$-system polarizations track each other in the Stokes $Q-U$ plane, while the $\mathrm{TiO} \alpha$-system behaves as a separate, unmodulated component (Figure 1). The "B filter" measurements actually describe a seasonal loop in the $Q-U$ plane, with an amplitude of about $0.8 \%$ and a random position angle from year to year.

The WUPPE Astro-1 UV data show a dramatic rise in polarization to about $2 \%$ at $3000 \AA$, followed by a steep drop below $2900 \AA$ (Nordsieck et al. 1994). The MgII emission line at $2800 \AA$ is unpolarized. The UV upturn has the same position angle as the $\alpha$ system in the optical region. There are then two geometrically separate components. One component is responsible for the visible blue continuum and $\mathrm{TiO} \gamma$-system polarization 


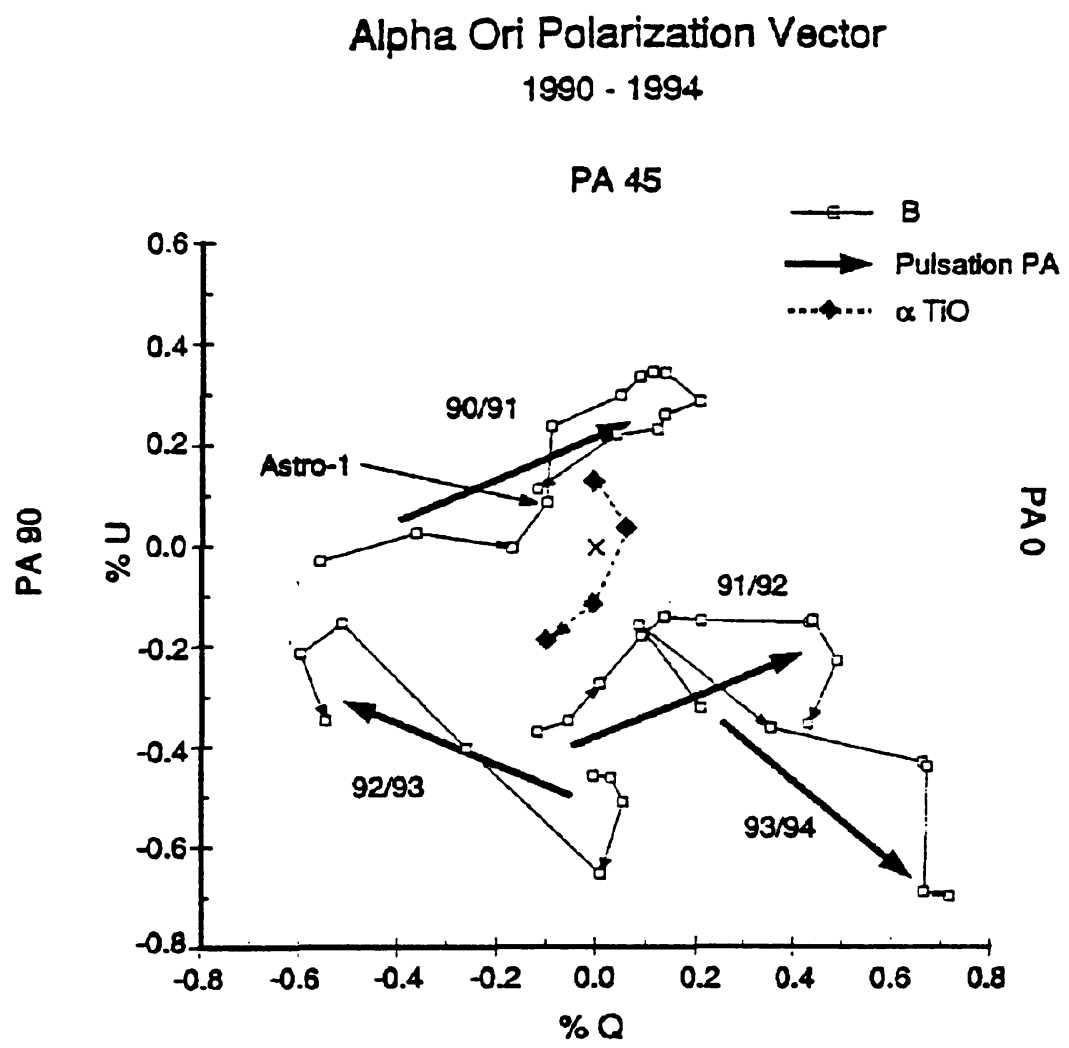

PA 135

Figure 1. Normalized $Q-U$ plot of $\alpha$ Ori data. The cross and "PA" indicate, respectively, the origin and position angle along the four directions shown.

and is modulated by a yearly cycle, albeit with a random position angle. The $\mathrm{TiO} \alpha$ system and UV upturn component seem to vary smoothly on a longer timescale. Other correlations confirm that the "B filter" component is indeed modulated by a radial pulsation.

The data indicate that the polarization is likely caused by photospheric Rayleigh scattering, with an asymmetry provided by a photospheric spot (Nordsieck et al. 1994). Non-radial pulsations (Holenstein 1993) could also originate in a hot region over the stellar disk. In the spot scenario, the polarization position angle would be parallel to the limb (see §2) and normal to the spot position angle. Remarkably, the UV Astro-2 WUPPE observations do indicate an average position angle very close to being $90^{\circ}$ apart from the position angle of the hot spot detected by Gilliland \& Dupree (1996) with the HST within a week of the Astro-2 flight in March 1995. 


\section{2. $\mathrm{V} \mathrm{CVn}$}

The remarkable semiregular variable V CVn (M4-6 IIIe, $P=192$ days) was one of the first objects noted to show large intrinsic polarization in broad-band data (Serkowski 1966). Most of the time V CVn shows a rather well behaved light curve and could be considered a small-amplitude Mira.

The PBO data for V CVn show that the polarization generally increases towards the violet, reaching at other times up to $10 \%$ (!) in that spectral region. The average polarization level is small when $\mathrm{V} \mathrm{CVn}$ is bright and vice versa. PBO data, as well as the earlier data (Coyne \& Magalhães $1977,1979)$ and in contrast to $\alpha$ Ori, show that the position angle is always observed to be within rather limited bounds, $\pm 15^{\circ}$ or so, over the years. Finally, the polarization generally decreases across $\mathrm{TiO}$ bands, sometimes with modest position angle changes.

The V CVn data are consistent with most of the optical polarization being produced in its photosphere. Some asymmetry exists already at the photospheric level, even though its nature is not completely clear. The stability of the position angle, as observed for decades now, raises the possibility that the asymmetry could be related to the rotation of the star. A modest rotation, for instance, could cause a noticeable pole-to-equator temperature variation over the stellar surface (Coyne \& Magalhães 1979).

\section{3. $o$ Cet}

The prototype of the Mira variable class is also known to be intrinsically polarized. The broad-band data of Shawl (1975) showed that the polarization of Mira in the blue increased sharply around phase 0.8 in the light curve, when the hydrogen lines from the periodic shock front first appear.

PBO data for Mira show that the overall polarization around maximum light (phases 0.9 through 0.3 ) is actually strongly related to the shock wave that progresses outward in each cycle. Also, the degree of polarization increases sharply through the Balmer emission lines (and Ca I $4226 \AA$; see Figure 2), as the latter appear and then fade, with little or no position angle rotation through the lines. $\mathrm{H} \gamma$ and $\mathrm{H} \delta$ reach their peak values earlier than $\mathrm{H} \beta$, the latter falling within a $\mathrm{TiO}$ band. The overall degree of polarization also rises and falls through the cycle, being steeper into the blue when the polarization is higher, around phase 0.0 . Changes in the polarization percentage and position angle across $\mathrm{TiO}$ bands are also seen.

These data suggest a non-spherically-symmetric shock front in Mira. The polarization then reflects the shock energy distribution, modified by the wavelength-dependent scattering and absorption opacities. Non-LTE effects, i.e. scattering in the lines (cf. Coyne \& Magalhães 1977 on V CVn), may be present. The fact that line radiation may have such an additional 


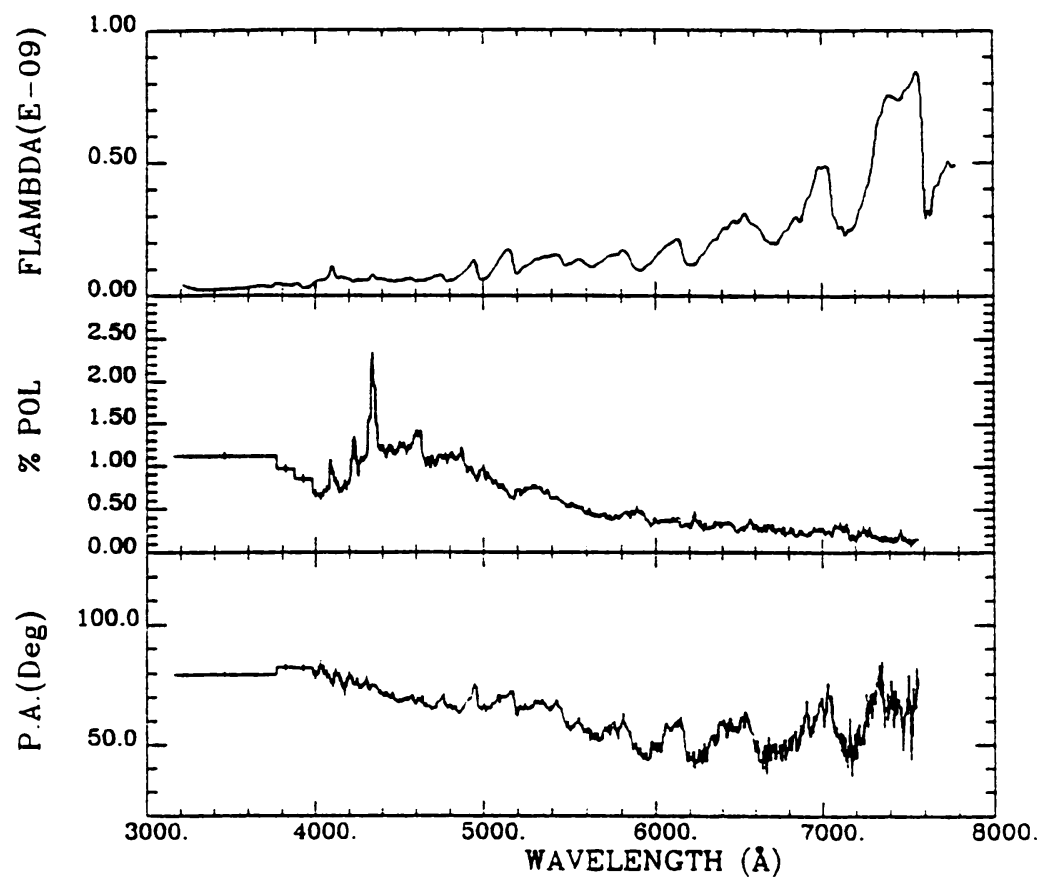

Figure 2. Flux, polarization and position angle for $o$ Ceti on September 27, 1990, around maximum light. Pine Bluff Observatory observations.

component besides (unpolarized) thermal recombination radiation indicates that polarization through the Balmer emission lines can be useful as a probe through the atmosphere.

\section{Final Remarks}

Spectropolarimetric monitoring data for almost $40 \mathrm{LRVs}$ now show that asymmetries in LRVs are indeed ubiquitous and that the phenomenon observed at later stages of stellar evolution (OH/IR stars, PPN, PN, etc.) appears early in the AGB and red supergiant phases.

Radiative transfer of polarized radiation will be useful to fully analyze the expected limb darkening and polarization from an LRV photosphere. Such an analysis could be done using the results from model atmospheres such as those by Plez, Brett \& Nordlund (1992). Comparison with the observations should provide important feedback to the model atmospheres themselves and to details such as $\mathrm{TiO}$ band formation. Monte Carlo scattering models (Lefevre \& Daniel 1988; Holenstein 1993; Rodrigues \& Magalhães 1995) have become sophisticated enough that modelling is already possible when scattering in an extended atmosphere and a combina- 
tion of geometries and processes (e.g., molecular, dust and atomic resonant scattering) are involved.

Finally, future coordination between polarimetric observations and techniques such as optical interferometry and radio imaging, for selected objects, may prove instrumental for gaining a complete picture of the asymmetries around LRVs and improved knowledge of the mass-loss process.

$\mathrm{AMM}$ is greatly indebted to the Brazilian agency $\mathrm{CNPq}$ and the $\mathrm{SOC}$ for supporting his attendance at the conference. Research with HPOL is supported by NASA contract NAS5-26777 with the University of Wisconsin. Polarimetry at the University of São Paulo is supported by FAPESP.

\section{References}

Bedijn, P. J. 1988, $A \& A, 205,105$

Bowen, G. H. \& Willson, L. A. 1991, ApJ, 375, L53

Chapman, J. M. 1994. Southern Stars, 35, 241

Chapman, J. M., Sivagnanam, P., Cohen, R. J. \& Le Squeren, A.M. 1994, MNRAS, 268, 475

Collison, A. J. \& Fix, J. D. 1992, ApJ, 390, 191

Coyne, G. V. \& Magalhães, A. M. 1977, $A J, 82,90$

Coyne, G. V. \& Magalhães, A. M. 1979, AJ, 84, 1200

Diamond, P. J., Kemball, A. J., Junor, W., Zensus, A., Benson, J. \& Dhawan, V.' 1994, $A p J, 430, \mathrm{~L} 61$

Gehrz, R. D. 1989, in IAU Symp. 135: Interstellar Dust, ed. L. J. Allamandola and A.G.G.M. Tielens (Kluwer), p. 445.

Greenhill, L.J., Colomer, F., Moran, J.M., Backer, D.C., Danchi, W.C. \& Bester, M. $1995, A p J, 449,365$

Gilliland, R. L. \& Dupree, A. K. 1996, ApJ, 463, L29

Harrington, J.P. 1969, Astrophys. Lett., 3, 165

Holenstein, B. D. 1993, PASP, 105, 322.

Johnson, J. J. \& Jones, T. J. 1991, AJ, 101, 1735

Kruszewski, A., Gehrels, T. \& Serkowski, K. 1968, AJ, 73, 677

Kwok, S. 1993, Ann. Rev. Astron. Astrophys., 31, 63

Lefevre, J. \& Daniel, J.-Y. 1988, in Polarized Radiation of Circumstellar Origin, ed. G. V. Coyne et al. (Vatican Obs.), p. 523

Magalhães, A. M. 1988, in Polarized Radiation of Circumstellar Origin, ed. G. V. Coyne et al. (Vatican Obs.), p. 461

Magalhães, A. M., Coyne, G. V. \& Benedetti, E. K. 1986, AJ, 91, 919

Nordsieck K. H. et al. 1994, BAAS, 26, 864

Plez, B., Brett, J. M. \& Nordlund, A. 1992, $A \& A, 256,551$

Plez, B. \& Lambert, D. L. 1994, ApJ, 425, L101

Rodrigues, C. V. \& Magalhães, A.M. 1995, in IAU Symp. 163: Wolf-Rayet Stars: Binaries, Colliding Winds, Evolution, ed. K. A. van der Hucht and P.M. Williams (Kluwer), p. 260

Schwarzschild, M. 1975, ApJ, 195, 137

Serkowski, K. 1966, ApJ, 144, 857

Shawl, S. J. 1975, $A J, 80,602$

Trammell, S. R., Dinerstein, H. L. \& Goodrich. R. W. 1994, AJ, 108, 984

Willson, L. A. \& Bowen, G. H. 1988, in Polarized Radiation of Circumstellar Origin, ed. G. V. Coyne et al. (Vatican Obs.), p. 485 